HEALTHCARE DELIVERY

\title{
Purpose-orientated stocking of procedure trolleys saves time in busy emergency centres
}

\author{
P Furstenburg, MB ChB, Dip PEC (SA); A Oosthuizen, MB ChB, Dip PEC (SA), FCEM, MMed; L Wallis, MB ChB, MD, FRCEM, FCEM (SA)
}

Division of Emergency Medicine, Faculty of Health Sciences, University of Cape Town, South Africa

Corresponding author: P Furstenburg (furstenburgphillip@gmail.com)

\begin{abstract}
Background. Inefficient storage and sourcing of routinely required consumables located on procedure trolleys result in time wasted when preparing for common procedures in emergency centres (ECs), contributing to poor efficiency and quality of care.

Objectives. We designed a novel purpose-orientated procedure trolley and evaluated its impact on time spent on procedure preparation and efficiency.

Methods. In an urban EC, eight participants were measured each day over 24 days, once using the standard setup and once using the modified procedure setup. During each simulation, efficiency markers were assessed (time spent on procedure preparation, steps taken, stops made, and time spent opening drawers to locate required items).

Results. The mean (standard deviation) time required to collect the required items for intravenous cannulation and blood sampling from the purpose-orientated trolley was 22.7 (3.66) seconds, compared with 49.2 (15.45) seconds using the standard trolley. There was a significant difference between the two trolleys in mean collection time $(p<0.0005)$ and in all the other categories: steps taken, stops made and drawer opening $(p<0.0005)$.

Conclusions. In our setting, stocking procedure trolleys in a purpose-orientated manner has the potential to improve efficiency by reducing time spent on procedure preparation.
\end{abstract}

S Afr Med J 2018;108(12):1024-1026. DOI:10.7196/SAMJ.2018.v108i12.13422

Emergency centres (ECs) worldwide are busy and stressful work environments ${ }^{[1]}$ In today's rapidly changing healthcare environment, ECs are expected to continuously improve the quality of care delivered to an expanding population of patients. ${ }^{[2]}$ This is also true in underresourced settings such as public facilities in South Africa (SA), where high patient volumes and acuity combine with overworked staff to lead to multiple quality challenges. ${ }^{[1]}$ One of the most visible challenges relates to long waiting times, a common cause of patient dissatisfaction. In addressing waiting times, numerous strategies are required, including more efficient use of existing resources. Common tasks routinely performed in ECs that consume unnecessary time and resources without improving service delivery result in inefficient workflow and have been identified as an area of concern (Van der Merwe LH, Viljoen VL, 'Trolley folly: Improving efficiency of retrieving equipment for commonly performed procedures in the EC': unpublished final-year MB ChB project, Department of Community Health, Stellenbosch University, 2016). Making small changes in workspace layout and workflow could produce significant efficiencies and reduce waiting times. ${ }^{[3]}$

There is growing interest in increasing workflow efficiency in healthcare through processes such as Lean thinking. ${ }^{[4]}$ Lean is a set of principles and techniques that drive organisations to continually add value to the product they deliver by enhancing process steps that are necessary, relevant and valuable, while eliminating those that fail to add value. ${ }^{[5-7]}$ Lean has been used in manufacturing for decades, and has been associated with enhanced product quality and overall corporate success. ${ }^{[5]}$ Lean thinking begins with driving out waste so that all work adds value and serves the client's needs. ${ }^{[2,5]}$ Identifying value-added and non-value-added steps in every process is the beginning of the journey towards Lean operations. ${ }^{[5]}$ Substantial improvements in waiting times and patient outcomes have been achieved and sustained following Lean thinking-inspired changes to employee roles, staffing and scheduling, communication, workspace layout and problem solving. ${ }^{[4]}$

Lean practices can be successfully achieved in emergency care, and can help healthcare providers be more productive, engaged and satisfied while enabling patients to receive the quality of care they want and expect. ${ }^{[2]}$ Numerous EC processes can be improved through Lean thinking, such as separate patient streams, structural changes, staffing changes and reorganisation of physical space; a recent review found that patient care improved after the implementation of Lean, with decreases in waiting times, length of stay, and proportion of patients leaving the $\mathrm{EC}$ without being seen. ${ }^{[4]}$

A high proportion of EC patients need medical procedures, from intravenous (IV) line placement to lumbar puncture. Inefficient storage and sourcing of routinely required consumables located on procedure trolleys result in time wasted when preparing for procedures, and this effect is magnified for tasks performed multiple times each day. ${ }^{[2]}$ Procedure trolleys are clinical resource trolleys equipped with the essential equipment necessary to perform specific procedures in the EC. Following the principles of Lean thinking, a simple intervention that changes the way these trolleys are stocked may improve efficiency and reduce time wastage, thereby freeing staff to spend that time on activities that add more value. Reorganisation of procedure trolleys can reduce errors and improve efficiency. ${ }^{[8-10]}$

\section{Objectives}

To investigate the effect on procedure preparation time of stocking a procedure trolley in a purpose-orientated manner. Secondary objectives included the number of steps taken, stops made, and number of times participants had to open a drawer during item collection. 


\section{Methods \\ Study design}

This was a quasi-experimental design testing the standard procedure trolley setup against a purpose-orientated setup.

\section{Study setting and population}

The study took place at Karl Bremer Hospital, an urban district hospital in Cape Town, SA. Data were collected during a simulated procedure in the EC. The participants were eight permanent full-time EC doctors, calculated with $80 \%$ power, an assumed effect size of 1.5 , and an approximate standard deviation (SD) of 15 seconds in each group. All participants gave signed informed consent. Ethics approval was granted by the hospital's Human Research Ethics Committee (ref. no. 656/2017) and facility approval by the National Health Research Database (ref. no. WC_201709_021).

\section{Data collection}

Data were collected over a period of 24 days, including weekends, with measurements taken once a day. The lead investigator measured time taken to search for and collect the items required for IV cannulation and blood sampling (Table 1) and take them to a specified area in the EC. Time to perform the task was recorded with the same stopwatch for all measurements. One doctor was measured each day, once using the standard setup and once using the modified procedure setup, and each doctor was measured six times in total.

The purpose-orientated trolley was stocked by the lead investigator and positioned at the same place where the standard procedure trolley is situated in everyday use. The order of setup was randomised to reduce the risk of bias from a repeated-measures effect. We collected 24 controls and 24 cases.

\section{Statistical analysis}

Descriptive statistics were presented for all variables. Categorical variables were reported as proportions with 95\% confidence intervals. The data reflected the amount of time spent, steps taken, stops made and drawer openings made while gathering necessary equipment during procedure preparation when using the standard EC consumables setup v. the purpose-orientated consumables setup.

Table 1. List of items required for intravenous cannulation and blood sampling
1. Tourniquet
2. Alcohol swab
3. Intravenous catheter
4. Syringe
5. Saline lock
6. EDTA vacutainer
7. Transparent film dressing
EDTA $=$ ethylenediaminetetra-acetic acid

Figures were used to illustrate the important findings from the study. The statistical analysis tried to identify whether a difference between the mean in the two groups existed by using a $t$-test. A post hoc power analysis was conducted to show whether adequate power existed for inferences to be acceptable. Where power lacked, preference was given for non-parametric analysis, such as the Wilcoxon rank-sum or Mann-Whitney test.

\section{Results}

Overall, the purpose-orientated trolley resulted in significant efficiency savings (Table 2 and Figs $1-3$ ).

The mean (SD) time to collect the required items for IV cannulation and blood sampling from the purpose-orientated trolley was 22.7 (3.66) seconds, compared with 49.2 (15.45) seconds using the standard trolley $(p<0.0005)$. The mean $(\mathrm{SD})$ number of steps was significantly reduced (17 (1) v. $28(13) ; p<0.0005)$, as were both the mean (SD) number of stops made (1 (0) v. $4(1) ; p<0.0005)$ and

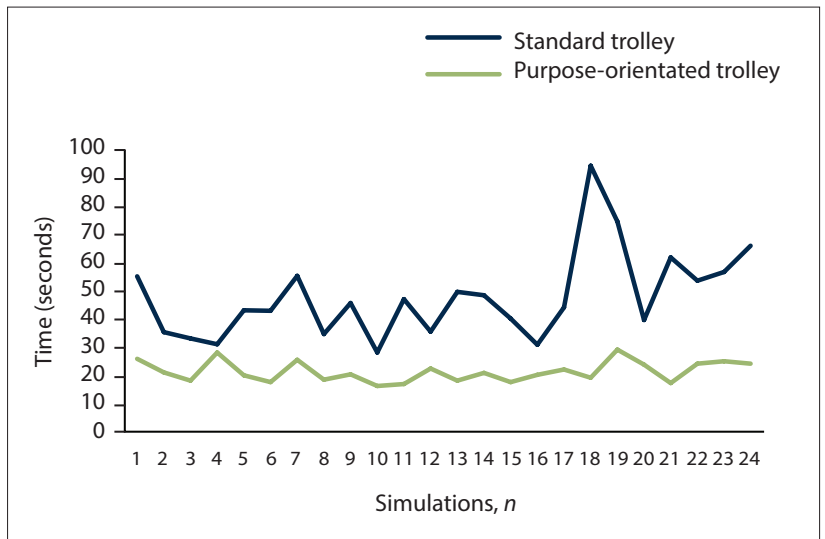

Fig. 1. Collection time of emergency instruments and material using standard trolley $v$. purpose-orientated trolley.

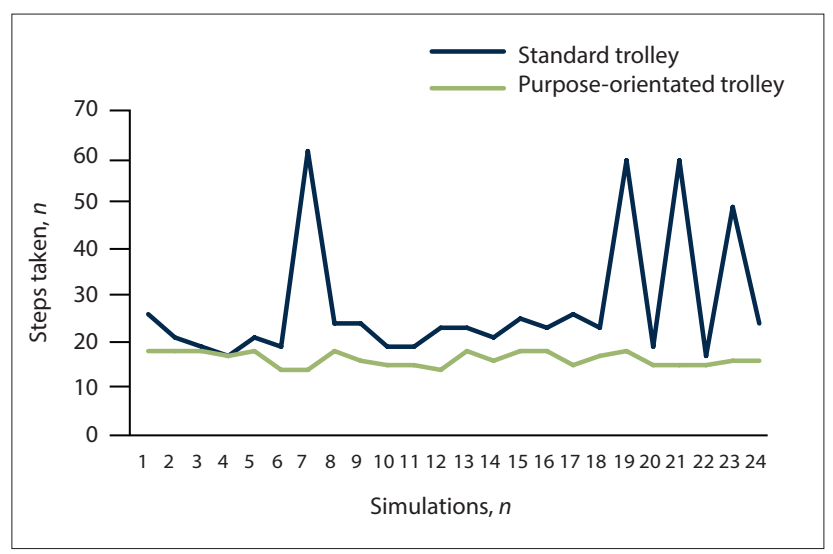

Fig. 2. Steps taken to collect emergency instruments and material from standard trolley $v$. purpose-orientated trolley.

Table 2. Efficiency indicators for standard trolley and purpose-orientated trolley

\begin{tabular}{llll}
\hline & \multicolumn{2}{c}{ Mean (SD), range } \\
\cline { 2 - 3 } & Standard trolley & Purpose-orientated trolley & $p$-value \\
\hline Time (seconds) & $49.2(15.45), 29.5-95.8$ & $22.7(3.66), 17.6-30.5$ & $<0.0005$ \\
Steps & $28(13), 18-62$ & $17(1), 15-19$ & $<0.0005$ \\
Stops & $4(1), 3-7$ & $1(0), 1-1$ & $<0.0005$ \\
Opening a drawer & $4(1), 4-8$ & $0(0), 0-0$ & $<0.0005$ \\
SD = standard deviation. & & &
\end{tabular}




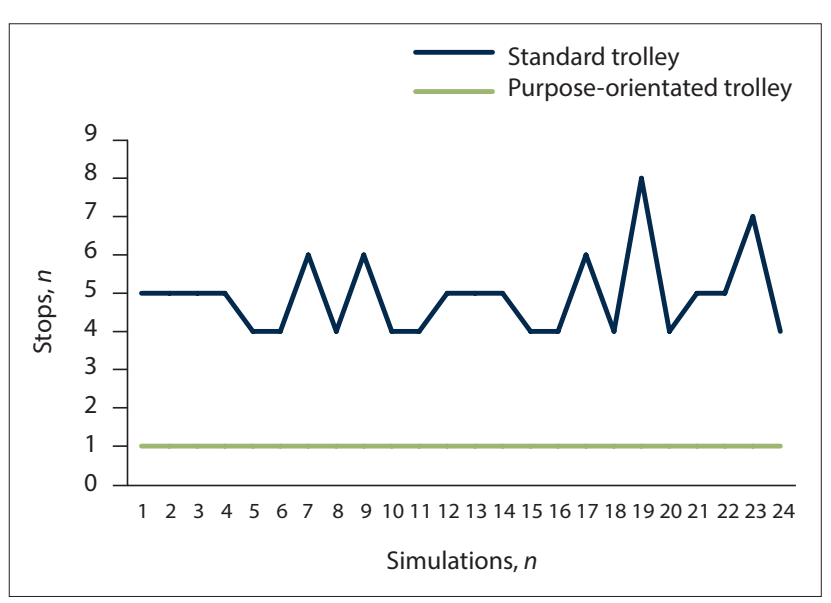

Fig. 3. Stops occurring during collecting emergency instruments and material from standard trolley $v$. purpose-orientated trolley.

the number of drawer openings to find equipment (4: there were no drawers on the procedure-orientated trolley).

\section{Discussion}

We demonstrated a significant reduction in time spent on procedure preparation when using a purpose-orientated trolley, as well as several other efficiencies. While a 26.5 -second reduction may not sound important, there are several considerations. Firstly, the EC at Karl Bremer Hospital is a busy one, with a high-acuity patient load. It sees over 90 patients a day and admits $\sim 50 \%$ of cases. Assuming that only the admitted patients have IV lines or blood work done (which we know is an underestimate of the actual volume), that is 45 patients per day. A saving of 26.5 seconds per patient frees up 20 minutes of doctor time over the day. If similar small changes in workflow and efficiency are possible in other areas of the EC, significant cost savings can be made, allowing doctors to work on more value-added areas of direct patient care and reducing waiting times.

Other authors have had similar success after modifying their resuscitation trolleys: drugs and equipment were accessed significantly faster, ${ }^{[8,9]}$ and IV preparation time was cut from 180 to 90 seconds. ${ }^{[10]}$ Time is not the only important factor, however. Reducing the number of steps taken may mean that staff will be less tired during their shifts, while eliminating drawer opening not only increases efficiency but reduces overall staff frustration. Other authors have shown similar results, e.g. by keeping tongue blades, ear speculums and otoscopes in the same place (Rutman et al. ${ }^{[10]}$ ). This is a simplistic but illustrative example of Lean in action: if a healthcare provider sees 20 patients on a shift and does not need to repeatedly search for these items, or to cross the room to gather supplies each time they are needed, he or she can save a couple of minutes with each encounter, adding value to patients and increasing EC capacity.

Only one participant was measured per day, once using the standard setup and once using the modified procedure setup, thus reducing the risk of bias from a repeated-measures effect. This study took place at a single unit, which may limit the external validity of our findings, but we believe that this site is typical of most large district hospitals in the country.

Our trolley redesign proved to be successful and did indeed increase efficiency by reducing time spent on procedure preparation. It is a simple example of how the implementation of Lean principles can improve healthcare. Our study reiterated the fact that, although Lean represents a fundamental change in the way we think of delivering care, it does not require an input of resources, and the specific resource changes made can be simple, small modifications specific to a specific clinical setting. ${ }^{[5]}$ This study can serve as a pilot for future work on small changes with incremental efficiency gains in busy ECs across the country.

\section{Conclusions}

By applying Lean principles to the design of the procedure trolley, we produced significant reductions in time taken to prepare for IV cannulation and blood work in a busy urban emergency centre. Similar efficiency gains in other areas of EC work can compound this work, leading to overall improved efficiency and reduced waiting times.

\section{Declaration. None.}

Acknowledgements. We acknowledge the help of Dr Dirk Smith, who assisted with data analysis.

Author contributions. PF and LW were the principal investigators and AO the research supervisor.

Funding. None.

Conflicts of interest. None.

1. Health24. 26 SA medical stats. 28 September 2012. http://www.health24.com/Medical-schemes/ News/26-SA-medical-stats-20130210 (accessed 31 October 2018).

2. Rutman L, Stone K, Reid J, Woodward GA, Migita R. Improving patient flow using Lean methodology: An emergency medicine experience. Curr Treat Options Pediatr 2015;1(4):359-371. https://doi. org/10.1007/s40746-015-0038-0

3. Grout JR. Mistake proofing: Changing designs to reduce error. Qual Saf Health Care 2006;15(1):42-44. https://doi.org/10.1136/qshc.2005.016030

4. Holden RJ. Lean thinking in emergency departments: A critical review. Ann Emerg Med 2011;57(3):265-278. https://www.ncbi.nlm.nih.gov/pubmed/21035904 (accessed 20 April 2016). https://doi.org/10.1016/j.annemergmed.2010.08.001

https://doi.org/10.1016/j.annemergmed.2010.08.001
5. Dickson EW, Singh S, Cheung DS, Wyatt CC, Nugent AS. Application of Lean manufacturing techniques in the emergency department. J Emerg Med 2009;37(2):177-182. https://www.ncbi.nlm.nih.gov/ pubmed/18722732 (accessed 20 November 2017). https://doi.org/10.1016/j.jemermed.2007.11.108

6. Ohno T, Bodek N. Toyota Production System: Beyond Large Scale Production. Portland, Ore. CRC Press, 1988. https://upbook.download/cloud/free-download-online-toyota-production-systembeyond-large-scale-production-pdf-by-taiichi-ohno-norman-bodek.html (accessed 4 January 2017). 7. Deming WE. The New Economics for Industry, Government \& Education. 2nd ed. Cambridge, Mass. Massachusetts Institute of Technology, 1993.

Donchin Y. Resuscitation trolleys: Human factors engineering. BMJ Qual Saf 2002;11(4):393. https:// doi.org/10.1136/qhc.11.4.393

9. Walker ST, Brett SJ, McKay A, Aggarwal R, Vincent C. The 'resus:station': The use of clinical simulations in a randomised crossover study to evaluate a novel resuscitation trolley. Resuscitation 2012;83(11):1374-1380. https://doi.org/10.1016/j.resuscitation.2012.06.026

10. Rutman LE, Migita R, Woodward GA, Klein EJ. Creating a leaner pediatric emergency department How rapid design and testing of a front-end model led to decreased wait time. Pediatr Emerg Care 2015;31(6):395-398. https://doi.org/10.1097/pec.0000000000000455 